\title{
Depth in an Arrangement of Hyperplanes
}

\author{
P. J. Rousseeuw and M. Hubert \\ Department of Mathematics and Computer Science, U.I.A., \\ Universiteitsplein 1, B-2610 Antwerp, Belgium \\ Peter.Rousseeuw@uia.ua.ac.be
}

\begin{abstract}
A collection of $n$ hyperplanes in $\mathbb{R}^{d}$ forms a hyperplane arrangement. The depth of a point $\theta \in \mathbb{R}^{d}$ is the smallest number of hyperplanes crossed by any ray emanating from $\theta$. For $d=2$ we prove that there always exists a point $\theta$ with depth at least $\lceil n / 3\rceil$. For higher dimensions we conjecture that the maximal depth is at least $\lceil n /(d+1)\rceil$. For arrangements in general position, an upper bound on the maximal depth is also established. Finally, we discuss algorithms to compute points with maximal depth.
\end{abstract}

\section{Definition of Depth}

Throughout this paper we consider a hyperplane arrangement $\mathcal{A}\left(H_{n}\right)$ in $\mathbb{R}^{d}$, where $H_{n}$ is a set of $n$ hyperplanes $h_{i} \subset \mathbb{R}^{d}$ for $i=1, \ldots, n$. For brevity, we often denote $\mathcal{A}\left(H_{n}\right)$ as $\mathcal{A}_{n}$. We say that a point $\boldsymbol{\theta} \in \mathbb{R}^{d}$ belongs to the exterior of $\mathcal{A}_{n}$ iff there exists a direction $\mathbf{u} \neq \mathbf{0}$ such that $\operatorname{ray}_{\theta}(\mathbf{u})=\{\boldsymbol{\theta}+\lambda \mathbf{u} ; \lambda \geq 0\}$ does not cross any $h_{i}$. Here we adopt the convention that parallels cross at infinity. For instance, the points $\boldsymbol{\eta}$ and $\boldsymbol{\tau}$ in Fig. 1 belong to the exterior of $\mathcal{A}_{n}$, but $\boldsymbol{\xi}$ does not because lines 2 and 3 are parallel.

Definition 1. The (arrangement) depth of a point $\theta \in \mathbb{R}^{d}$ relative to $\mathcal{A}_{n}$ is defined as the smallest number of hyperplanes $h_{i}$ that need to be removed to set $\boldsymbol{\theta}$ free (i.e., so that it lies in the exterior of the remaining hyperplanes). Equivalently, it is the smallest number of hyperplanes crossed (possibly at infinity) by any $\operatorname{ray}_{\theta}(\mathbf{u})$ with $\mathbf{u} \neq \mathbf{0}$.

For any vector $\mathbf{u} \neq \mathbf{0}$ we denote by $\operatorname{ncross}\left(\boldsymbol{\theta}, \mathbf{u}, \mathcal{A}_{n}\right)$ the number of hyperplanes crossed (possibly at infinity) by $\operatorname{ray}_{\theta}(\mathbf{u})$. Therefore

$$
\operatorname{depth}\left(\boldsymbol{\theta}, \mathcal{A}_{n}\right)=\min _{\mathbf{u} \neq \mathbf{0}} \operatorname{ncross}\left(\boldsymbol{\theta}, \mathbf{u}, \mathcal{A}_{n}\right)
$$




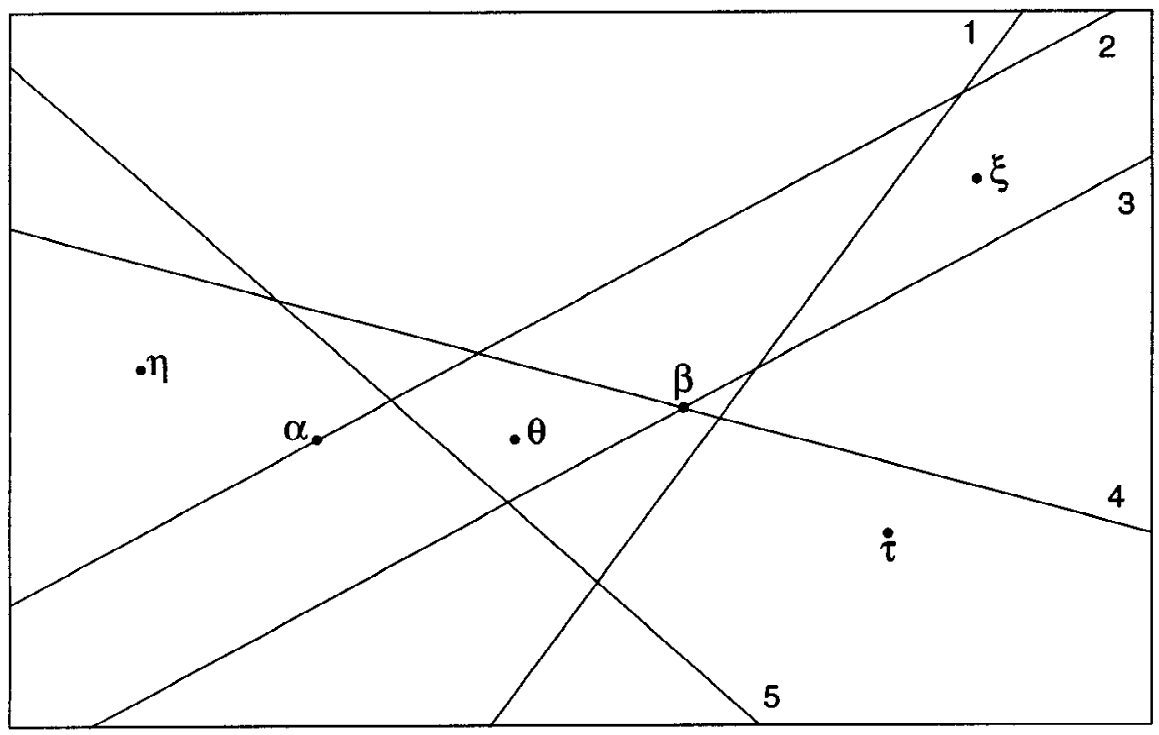

Fig. 1. An arrangement $\mathcal{A}_{5}$ of five hyperplanes (lines) in $\mathbb{R}^{2}$. The points $\eta$ and $\tau$ belong to the exterior of $\mathcal{A}_{5}$, the points $\alpha$ and $\xi$ have depth 1 , whereas $\operatorname{depth}\left(\theta, \mathcal{A}_{5}\right)=2$ and $\operatorname{depth}\left(\beta, \mathcal{A}_{5}\right)=3$.

Equivalently let the minimum in (1.1) range over all $\|\mathbf{u}\|=1$ where $\mathbf{u}$ is not parallel to any hyperplane in $\mathcal{A}_{n}$ since slightly rotating a parallel $\mathbf{u}$ decreases $n \operatorname{cross}\left(\boldsymbol{\theta}, \mathbf{u}, \mathcal{A}_{n}\right)$. (In fact, one could even restrict $\mathbf{u}$ to a finite set of directions "in between" the hyperplanes.)

Consider again Fig. 1. We see that any $\operatorname{ray}_{\theta}(\mathbf{u})$ crosses at least two lines, hence $\operatorname{depth}\left(\boldsymbol{\theta}, \mathcal{A}_{5}\right)=2$. Equivalently, we could remove lines 1 and 3 so that $\boldsymbol{\theta}$ belongs to the exterior of the arrangement formed by the remaining lines 2, 4, and 5. Moreover, $\operatorname{depth}\left(\boldsymbol{\alpha}, \mathcal{A}_{5}\right)=\operatorname{depth}\left(\boldsymbol{\xi}, \mathcal{A}_{5}\right)=1$ since we only have to remove line 2 to set $\boldsymbol{\alpha}$ and $\boldsymbol{\xi}$ free. Finally, $\operatorname{depth}\left(\boldsymbol{\beta}, \mathcal{A}_{5}\right)=3$ because $\boldsymbol{\beta}$ lies on lines 3 and 4 , and to set $\boldsymbol{\beta}$ free we also have to remove one of the lines $\{1,2,5\}$ which form a triangle around $\boldsymbol{\beta}$.

Remark 1. The definition of the arrangement depth originates from the concept of the regression depth in statistics, introduced in Rousseeuw and Hubert (1999). The goal of linear regression analysis is to fit a data set $Z_{n}=\left\{\left(x_{i 1}, \ldots, x_{i, d-1}, y_{i}\right) ; i=1, \ldots, n\right\} \subset$ $\mathbb{R}^{d}$ by a hyperplane

$$
y=\theta_{1} x_{1}+\cdots+\theta_{d-1} x_{d-1}+\theta_{d},
$$

where the fit parameters are combined in a vector $\boldsymbol{\theta}=\left(\theta_{1}, \ldots, \theta_{d}\right)$. This problem is dualized as follows. The dual space is the set of all possible fit vectors $\boldsymbol{\theta}$, hence it is $\left\{\left(\theta_{1}, \ldots, \theta_{d}\right) ;\right.$ all $\left.\theta_{j} \in \mathbb{R}\right\}=\mathbb{R}^{d}$. Moreover, each data point $\mathbf{z}_{i}=\left(x_{i 1}, \ldots, x_{i, d-1}, y_{i}\right)$ is mapped to the set $D\left(\mathbf{z}_{i}\right)$ of all $\boldsymbol{\theta}$ that pass through $\mathbf{z}_{i}$, so $D\left(\mathbf{z}_{i}\right)$ is the hyperplane $h_{i}$ given by

$$
h_{i}=D\left(\mathbf{z}_{i}\right)=\left\{\left(\theta_{1}, \ldots, \theta_{d}\right) ; \theta_{d}=-x_{i 1} \theta_{1}-\cdots-x_{i, d-1} \theta_{d-1}+y_{i}\right\}
$$


The regression depth of any fit $\boldsymbol{\theta}$ coincides with the depth of the point $\boldsymbol{\theta}$ in the arrangement $\mathcal{A}_{n}:=\left\{h_{i} ; i=1, \ldots, n\right\}$ in the sense of Definition 1 above.

Remark 2. In the definition of the arrangement depth there are no preferential directions, so this notion is isotropic. One way to see this is by noting that our depth is invariant under rotations (in fact, even under all nonsingular affine transformations). If on the other hand we restrict ourselves to vertical directions $\mathbf{u}$ in Definition 1, we would obtain the levels of the arrangement (see, e.g., Chapter 3 in Edelsbrunner (1987)).

Remark 3. Although the arrangement depth has not been studied before, a notion of the depth of a point $\mathbf{p} \in \mathbb{R}^{d}$ relative to a configuration of points $\left\{\mathbf{x}_{1}, \ldots, \mathbf{x}_{n}\right\} \subset \mathbb{R}^{d}$ does exist. In statistics this is called the location depth (Tukey, 1975; Donoho and Gasko, 1992; Rousseeuw and Ruts, 1996). The location depth of $\mathbf{p}$ is the smallest number of data points $\mathbf{x}_{i}$ that lie in any closed halfspace with boundary through $\mathbf{p}$. Although the location depth is quite different from the arrangement depth, some of their properties are similar, e.g., it is also isotropic.

Remark 4. The notion of arrangement depth could also be used in the setting of projective space. There our convention that parallel lines cross at infinity would no longer be needed.

\section{Maximal Depth for $d \leq 2$}

We are interested in the highest depth that can be attained relative to a given arrangement $\mathcal{A}_{n}$ in $\mathbb{R}^{d}$. Formally, this is the integer

$$
\max _{\boldsymbol{\theta}} \operatorname{depth}\left(\boldsymbol{\theta}, \mathcal{A}_{n}\right)
$$

where $\boldsymbol{\theta}$ ranges over $\mathbb{R}^{d}$. In fact, we can easily see (e.g., by looking at Fig. 1) that it suffices to let $\boldsymbol{\theta}$ range over all intersection points of $d$ hyperplanes. Note that (2.1) is at most $n$, which can only be attained when all $n$ hyperplanes are concurrent (then we can set $\boldsymbol{\theta}$ equal to their common intersection point, hence $\left.\operatorname{depth}\left(\boldsymbol{\theta}, \mathcal{A}_{n}\right)=n\right)$.

In general, there may be more than one $\boldsymbol{\theta}$ with maximal depth. Intuitively, a point $\boldsymbol{\theta}$ attaining (2.1) can be seen as a "deepest point" or a "most central point." The higher $\operatorname{depth}\left(\boldsymbol{\theta}, \mathcal{A}_{n}\right)$ the more "central" $\boldsymbol{\theta}$ is. Conversely, points with the lowest possible depth (namely zero) lie in the exterior of $\mathcal{A}_{n}$.

When $d=1$ the arrangement $\mathcal{A}_{n}$ is a set $\left\{h_{1}, \ldots, h_{n}\right\}$ of real numbers. Suppose without loss of generality that $h_{1} \leq h_{2} \leq \cdots \leq h_{n}$ (note that some $h_{i}$ may coincide). When $n$ is odd we define the median set as the singleton $\left\{h_{\lceil n / 2\rceil}\right\}$ where the ceiling $\lceil\lambda\rceil$ denotes the smallest integer $\geq \lambda$. When $n$ is even we define the median set as the closed segment $\left[h_{n / 2}, h_{(n / 2)+1}\right]$. In either case, the maximal depth (2.1) is attained at any $\theta$ in the median set, hence

$$
\max _{\theta} \operatorname{depth}\left(\theta, \mathcal{A}_{n}\right) \geq\left\lceil\frac{n}{2}\right\rceil
$$


for $d=1$. The lower bound $\lceil n / 2\rceil$ is sharp (e.g., consider the case where $h_{1}<h_{2}<$ $\left.\cdots<h_{n}\right)$. When several $h_{i}$ coincide, the maximal depth can become strictly larger than $\lceil n / 2\rceil$.

We now consider the case $d=2$, where the hyperplanes become lines $l_{i}$ as in Fig. 1 . We will show that any arrangement of lines in $\mathbb{R}^{2}$ admits a point with depth $\lceil n / 3\rceil$ or higher. (In the sense that $\mathcal{A}\left(H_{n}\right)$ also contains all intersections formed by $H_{n}$ we can even say that each arrangement in $\mathbb{R}^{2}$ contains a point with depth at least $\lceil n / 3\rceil$.)

Theorem 1. For any arrangement $\mathcal{A}_{n}$ in $\mathbb{R}^{2}$ we have

$$
\max _{\boldsymbol{\theta}} \operatorname{depth}\left(\boldsymbol{\theta}, \mathcal{A}_{n}\right) \geq\left\lceil\frac{n}{3}\right\rceil \text {. }
$$

The lower bound $\lceil n / 3\rceil$ is sharp.

Proof. We may assume without loss of generality that none of the $l_{i}$ are vertical (otherwise, we can rotate $\mathcal{A}_{n}$ slightly). We start by ordering the $n$ lines $l_{i}$ by decreasing slopes. If $l_{i}$ and $l_{j}$ are parallel and $l_{i}$ lies above $l_{j}$ we put $l_{i}$ before $l_{j}$. (If $l_{i}=l_{j}$ it does not matter in which order we put them.) For example, the lines in Fig. 1 were ordered in this way. If $n=3 m$ or $n=3 m+1$ for some integer $m$, we denote by $A$ the set of the first $m$ lines, and by $C$ the set of the last $m$ lines. If $n=3 m+2$ we take $|A|=|C|=m+1$. The general formula is therefore $|A|=|C|=\lceil(n-1) / 3\rceil$. In each case, the set $B$ is formed by the remaining lines in between. In Fig. 1 we have $A=\left\{l_{1}, l_{2}\right\}, B=\left\{l_{3}\right\}$, and $C=\left\{l_{4}, l_{5}\right\}$.

We now consider the sets $H_{1}=A \cup B$ and $H_{2}=B \cup C$. Using the duality (1.2) we obtain two point sets $D^{-1}\left(H_{1}\right)$ and $D^{-1}\left(H_{2}\right)$ in $\mathbb{R}^{2}$. By the ham-sandwich theorem (see, e.g., Edelsbrunner (1987), page 69) there exists a line $L$ that simultaneously bisects $D^{-1}\left(H_{1}\right)$ and $D^{-1}\left(H_{2}\right)$. This means that both of the open halfplanes determined by $L$ contain at most $\left\lfloor\left|H_{1}\right| / 2\right\rfloor$ points of $D^{-1}\left(H_{1}\right)$ and at most $\left\lfloor\left|H_{2}\right| / 2\right\rfloor$ points of $D^{-1}\left(H_{2}\right)$. Note that $\left\lfloor\left|H_{1}\right| / 2\right\rfloor=\left\lfloor\left|H_{2}\right| / 2\right\rfloor=m$. (Here, the floor $\lfloor\lambda\rfloor$ is the largest integer $\leq \lambda$.) We may assume without loss of generality that $L$ is not vertical. Now put $\tilde{\boldsymbol{\theta}}:=\bar{D}(L)$ and denote

$$
\begin{aligned}
& (A \cup B)^{+}(\tilde{\boldsymbol{\theta}})=\left\{l_{i} \in A \cup B ; l_{i} \text { lies strictly above } \tilde{\boldsymbol{\theta}}\right\}, \\
& (A \cup B)^{-}(\tilde{\boldsymbol{\theta}})=\left\{l_{i} \in A \cup B ; l_{i} \text { lies strictly below } \tilde{\boldsymbol{\theta}}\right\}, \\
& (A \cup B)^{0}(\tilde{\boldsymbol{\theta}})=\left\{l_{i} \in A \cup B ; l_{i} \text { passes through } \tilde{\boldsymbol{\theta}}\right\},
\end{aligned}
$$

and analogously for $(B \cup C)^{+}(\tilde{\boldsymbol{\theta}}),(B \cup C)^{-}(\tilde{\boldsymbol{\theta}})$, and $(B \cup C)^{0}(\tilde{\boldsymbol{\theta}})$. By construction of $\tilde{\boldsymbol{\theta}}$ we know that $\left|(A \cup B)^{+}(\tilde{\boldsymbol{\theta}})\right| \leq m,\left|(A \cup B)^{-}(\tilde{\boldsymbol{\theta}})\right| \leq m,\left|(B \cup C)^{+}(\tilde{\boldsymbol{\theta}})\right| \leq m$, and $\mid(B \cup$ $C)^{-}(\tilde{\boldsymbol{\theta}}) \mid \leq m$.

We will now prove that $\operatorname{depth}\left(\tilde{\boldsymbol{\theta}}, \mathcal{A}_{n}\right) \geq\lceil n / 3\rceil$. For this we consider unit vectors $\mathbf{u}=\left(u_{1}, u_{2}\right)$ which we can write as $\mathbf{u}=\left(\cos \left(\alpha_{u}\right), \sin \left(\alpha_{u}\right)\right)$. We may assume without loss of generality that $u_{1}=\cos \left(\alpha_{u}\right) \neq 0$ because no $l_{i}$ is vertical. (For $\alpha_{u}= \pm \pi / 2$ we obtain the same $n \operatorname{cross}\left(\boldsymbol{\theta}, \mathbf{u}, \mathcal{A}_{n}\right)$ if we replace $\alpha_{u}$ by $\alpha_{u} \pm \varepsilon$ for $\varepsilon>0$ small enough.) Therefore $\tan \left(\alpha_{u}\right)=u_{2} / u_{1}$ exists.

We begin with the case where $\tan \left(\alpha_{u}\right)<\min _{l_{i} \in B} \operatorname{slope}\left(l_{i}\right)$. We first assume that $u_{1}>0$ as in Fig. 2(a). There the three lines $l_{3}, l_{7}$, and $l_{10}$ belong to $A \cup B$, and indeed the slope of $\mathbf{u}$ is lower than the slope of those lines. Then any line in $A \cup B$ strictly 


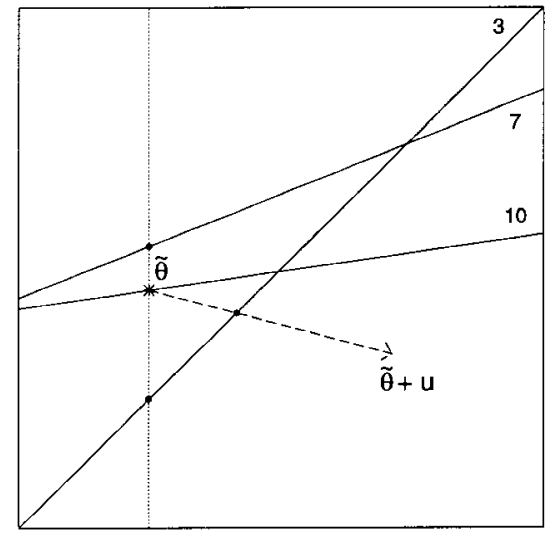

(a)

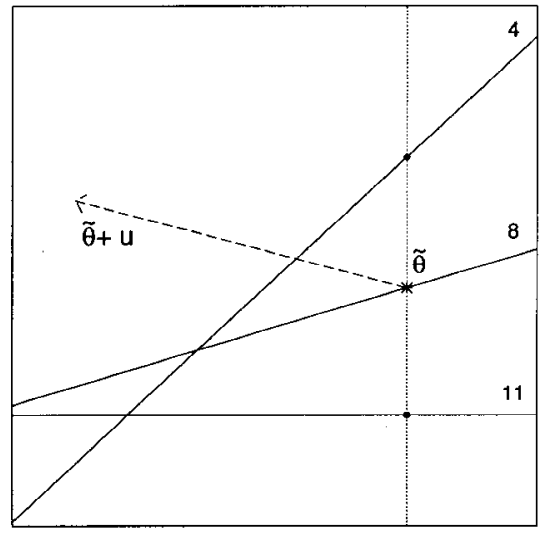

(b)

Fig. 2. Illustrations of the cases (a) $u_{1}>0$ and (b) $u_{1}<0$ in the proof of Theorem 1 .

below $\tilde{\boldsymbol{\theta}}$ (like $l_{3}$ ) $\operatorname{crosses}_{\operatorname{ray}_{\tilde{\theta}}}(\mathbf{u})$, whereas the lines in $A \cup B$ strictly above $\tilde{\boldsymbol{\theta}}$ (like $l_{7}$ ) do not $\operatorname{cross}_{\operatorname{ray}_{\tilde{\theta}}}(\mathbf{u})$. Also the lines in $A \cup B$ that pass through $\tilde{\boldsymbol{\theta}}$ itself (like $l_{10}$ ) obviously $\operatorname{cross}_{\tilde{\theta}}(\mathbf{u})$. By construction of $\tilde{\boldsymbol{\theta}}$ we know that $\left|(A \cup B)^{-}(\tilde{\boldsymbol{\theta}})\right| \leq m$, hence at least $|(A \cup B)|-m$ lines in $A \cup B$ cross ray $\tilde{\tilde{\theta}}(\mathbf{u})$. If $n=3 m$ we find $|A \cup B|-m=m=\lceil n / 3\rceil$, and for $n=3 m+1$ or $n=3 m+2$ we obtain $|A \cup B|-m=m+1=\lceil n / 3\rceil$. In each of these cases $n \operatorname{cross}\left(\tilde{\boldsymbol{\theta}}, \mathbf{u}, \mathcal{A}_{n}\right) \geq \operatorname{ncross}(\tilde{\boldsymbol{\theta}}, \mathbf{u}, A \cup B) \geq\lceil n / 3\rceil$.

Since $u_{1} \neq 0$ we are left with $u_{1}<0$ as in Fig. 2(b). This time a line in $A \cup B$ crosses $\operatorname{ray}_{\tilde{\theta}}(\mathbf{u})$ iff it lies strictly above $\tilde{\boldsymbol{\theta}}$ (like $\left.l_{4}\right)$, and using $\left|(A \cup B)^{+}(\tilde{\boldsymbol{\theta}})\right| \leq m$ we again find $n \operatorname{cross}\left(\tilde{\boldsymbol{\theta}}, \mathbf{u}, \mathcal{A}_{n}\right) \geq\lceil n / 3\rceil$.

In the case where $\tan \left(\alpha_{u}\right)>\max _{l_{i} \in B}$ slope $\left(l_{i}\right)$ we consider $B \cup C$, for which $\mid(B \cup$ $C)^{-}(\tilde{\boldsymbol{\theta}}) \mid \leq m$ and $\left|(B \cup C)^{+}(\tilde{\boldsymbol{\theta}})\right| \leq m$. Finally, when $\min _{l_{i} \in B} \operatorname{slope}\left(l_{i}\right) \leq \tan \left(\alpha_{u}\right) \leq$ $\max _{l_{i} \in B}$ slope $\left(l_{i}\right)$ we consider $A \cup C$ and use $\left|A^{-}(\tilde{\boldsymbol{\theta}})\right| \leq\left|(A \cup B)^{-}(\tilde{\boldsymbol{\theta}})\right| \leq m$ as well as $\left|C^{+}(\tilde{\boldsymbol{\theta}})\right| \leq\left|(B \cup C)^{+}(\tilde{\boldsymbol{\theta}})\right| \leq m$.

To see why the lower bound $\lceil n / 3\rceil$ in Theorem 1 is sharp, consider an arrangement $\mathcal{A}_{n}=\left\{l_{1}, \ldots, l_{n}\right\}$ where the lines $l_{i}$ are given by $\theta_{2}=-t_{i} \theta_{1}+t_{i}^{2}$ where $0<t_{1}<$ $\cdots<t_{n}<\infty$. The duals of these lines are $n$ different points on the moment curve $\left\{\left(t, t^{2}\right) ; 0<t<\infty\right\}$.

The proof of Theorem 1 constructs a point $\tilde{\boldsymbol{\theta}}$ whose depth is always at least $\lceil n / 3\rceil$. In highly unbalanced arrangements, such as the $\mathcal{A}_{n}$ based on the moment curve above, this is also the maximal depth. However, for $\mathcal{A}_{n}$ which are more symmetric the maximal depth becomes higher. In a sense, the maximal depth reflects how balanced $\mathcal{A}_{n}$ is. Note that the maximal depth (2.1) is invariant under affine transformations, and only depends on the combinatorial structure of $\mathcal{A}_{n}$ (as characterized by, say, its incidence graph).

Note that the proof of Theorem 1 yields a linear time algorithm to compute a point with depth at least $\lceil n / 3\rceil$. Indeed, the point $\tilde{\boldsymbol{\theta}}$ is obtained from a ham-sandwich cut, 
which can be constructed in $\mathrm{O}(n)$ time by the algorithm of Lo et al. (1994). Also note that we can translate the entire proof of Theorem 1 to the dual space. The ordering of the lines $l_{i}$ then corresponds to ordering the configuration of points $D\left(h_{i}\right)=\left(x_{i}, y_{i}\right)$ by their $x_{i}$. Then $A$ becomes the third of that configuration with the smallest $x$-coordinates, $C$ the third with the largest $x$-coordinates, and $B$ the rest. Carrying out a ham-sandwich cut on $A \cup B$ and $B \cup C$ then yields a line with equation $y=\tilde{\theta}_{1} x+\tilde{\theta}_{2}$.

Theorem 1 allows us to obtain a rather surprising result, which is a counterpart to Birch's (1959) result about a configuration of points in the plane.

Theorem 2. Consider $n=3 m$ lines in $\mathbb{R}^{2}$, all with distinct slopes. Then the $n$ lines can be partitioned into $m$ triplets $(i, j, k)$ so that the $m$ closed triangles $\triangle\left(l_{i}, l_{j}, l_{k}\right)$ have a nonempty intersection.

Proof. From Theorem 1 we know there exists a point $\tilde{\boldsymbol{\theta}}$ with $\operatorname{depth}\left(\tilde{\boldsymbol{\theta}}, \mathcal{A}_{n}\right) \geq m$. For each line $l_{i}$ we now consider the orthogonal projection $\boldsymbol{\xi}_{i}$ of $\tilde{\boldsymbol{\theta}}$ on $l_{i}$. Let $\alpha_{i}$ be the angle of $\boldsymbol{\xi}_{i}-\tilde{\boldsymbol{\theta}}$ (where $\boldsymbol{\xi}_{i}=\tilde{\boldsymbol{\theta}}$ may be assigned to any angle), and rank them to $0 \leq \alpha_{1}<\cdots<2 \pi$. Then take the triplets $(1, m+1,2 m+1),(2, m+2,2 m+2), \ldots,(m, 2 m, n)$. Each of these triplets yields a closed triangle containing $\tilde{\boldsymbol{\theta}}$. Otherwise there would be some $j$ such that the angles $\alpha_{j}, \ldots, \alpha_{j+m}$ range over more than $\pi$. (If some $j+k>n$ we put $\alpha_{j+k}:=\alpha_{j+k-n}+2 \pi$.) Then take $\alpha_{u}=\left(\alpha_{j}+\alpha_{j+m}\right) / 2$ inside that range, yielding $n \operatorname{cross}\left(\tilde{\boldsymbol{\theta}}, \mathbf{u}, \mathcal{A}_{n}\right) \leq m-1$ which contradicts $\operatorname{depth}\left(\tilde{\boldsymbol{\theta}}, \mathcal{A}_{n}\right) \geq m$.

If we want to dispense with the restrictions that $n$ is a multiple of 3 and that all slopes are different, we have to use more than triangles. In general we have a set $I$ of 1,2, 3 or more lines, whose slopes need not be distinct. We then put

$$
C(I)=\left\{\boldsymbol{\theta} \in \mathbb{R}^{2} ; \operatorname{depth}(\boldsymbol{\theta}, I) \geq 1\right\}
$$

which can be a line, the union of two lines, the union of three lines with the closed triangle between them, etc. For instance, when $|I|=3$ and two of the lines are parallel, $C(I)$ is the union of the remaining line with the closed strip formed by the two parallel lines. In general we could call $C(I)$ the "contractible hull" of $I$ because it is the smallest set without holes that encompasses $I$. We now obtain:

Theorem 3. Any set of $n$ lines in the plane can be partitioned into $k=\lceil n / 3\rceil$ subsets $I_{1}, \ldots, I_{k}$ such that $C\left(I_{1}\right), \ldots, C\left(I_{k}\right)$ have a nonempty intersection.

Proof. Let $\tilde{\boldsymbol{\theta}}$ be the point and let $A, B$, and $C$ be the sets constructed in the proof of Theorem 1 . We will prove that we can partition the $n$ lines $\left\{l_{i}\right\}$ into $k=\lceil n / 3\rceil$ subsets $I_{j}$ such that $\tilde{\boldsymbol{\theta}}$ belongs to each $C\left(I_{j}\right)$. If $n \neq 3 m$ both $|A \cup B|$ and $|B \cup C|$ are odd, so $\tilde{\boldsymbol{\theta}}$ will lie on at least one or two lines, denoted by $L=\left\{l_{1}\right\}$ or $L=\left\{l_{1}, l_{2}\right\}$. We may assume that $\tilde{\boldsymbol{\theta}}$ does not lie on any line $l_{i} \notin L$. Indeed, assume that a collection of lines $M$, distinct from $L$, passes through $\tilde{\boldsymbol{\theta}}$. Then it holds that $\tilde{\boldsymbol{\theta}} \in C\left(I_{j}\right)$ as soon as at least one line from $M$ belongs to $I_{j}$. If the theorem holds when all lines $l_{i} \notin L$ are strictly above/below $\tilde{\boldsymbol{\theta}}$, it will therefore remain true when some of these lines pass through $\tilde{\boldsymbol{\theta}}$. 
First consider the case that $n=3 m$. As in the proof of Theorem 1 we denote

$$
\begin{aligned}
& A^{+}(\tilde{\boldsymbol{\theta}})=\left\{l_{i} \in A ; l_{i} \text { lies strictly above } \tilde{\boldsymbol{\theta}}\right\}, \\
& A^{-}(\tilde{\boldsymbol{\theta}})=\left\{l_{i} \in A ; l_{i} \text { lies strictly below } \tilde{\boldsymbol{\theta}}\right\},
\end{aligned}
$$

and analogously for $B^{+}(\tilde{\boldsymbol{\theta}}), B^{-}(\tilde{\boldsymbol{\theta}}), C^{+}(\tilde{\boldsymbol{\theta}})$, and $C^{-}(\tilde{\boldsymbol{\theta}})$. If $\left|A^{+}\right|=q$ for some $0 \leq q \leq$ $m$, we know by construction of $\tilde{\boldsymbol{\theta}}$ that $\left|B^{-}\right|=\left|C^{+}\right|=q$ and $\left|A^{-}\right|=\left|B^{+}\right|=\left|C^{-}\right|=m-$ $q$. We can now partition $H_{n}$ into $q$ triplets $\left(l_{i}, l_{j}, l_{h}\right)$ with $l_{i} \in A^{+}, l_{j} \in B^{-}$, and $l_{h} \in C^{+}$ and $m-q$ triplets $\left(l_{i}, l_{j}, l_{h}\right)$ with $l_{i} \in A^{-}, l_{j} \in B^{+}$, and $l_{h} \in C^{-}$. Finally it is easy to see that $\tilde{\boldsymbol{\theta}} \in C\left(I_{k}\right)$ for each triplet $I_{k}$.

If $n=3 m+1$ we know that $\tilde{\boldsymbol{\theta}}$ lies on one line $l_{1} \in A \cup B$ and on one line $l_{2}$ in $B \cup C$. This implies that we must consider two different situations:

(a) $l_{1}=l_{2}$ and $l_{1} \in B$,

(b) $l_{1} \in A$ and $l_{2} \in C$.

(The case when $l_{1} \in A$ and $l_{2} \in B$ follows from (a) since this $\tilde{\boldsymbol{\theta}}$ lies on one more line than the $\tilde{\boldsymbol{\theta}}$ in (a). The case $l_{1} \in B$ and $l_{2} \in C$ is analogous.) First consider situation (a). Set $I_{1}=\left\{l_{1}\right\}$, hence $\tilde{\boldsymbol{\theta}} \in C\left(I_{1}\right)$. Furthermore, $|A|=\left|B \backslash\left\{l_{1}\right\}\right|=|C|=m$, so the situation is reduced to the case $n=3 m$. We can then partition $H_{n} \backslash\left\{l_{1}\right\}$ into $m$ triplets $I_{2}, \ldots, I_{m+1}$, each satisfying $\tilde{\boldsymbol{\theta}} \in C\left(I_{k}\right)$. In situation (b) we choose $I_{1}=\left\{l_{1}\right\}$ and $I_{2}=\left\{l_{2}\right\}$. If now $\left|A^{+}\right|=q$, then $\left|B^{-}\right|-1=\left|C^{+}\right|=q$ and $\left|A^{-}\right|=\left|B^{+}\right|-1=\left|C^{-}\right|=m-q-1$. This implies that we can partition $H_{n} \backslash\left\{l_{1}, l_{2}\right\}$ into $q$ triplets in $A^{+} \times B^{-} \times C^{+}$and $m-q-1$ triplets in $A^{-} \times B^{+} \times C^{-}$. Finally, the two remaining observations in $B$ may be assigned to any of the $m+1=\lceil n / 3\rceil$ subsets.

The final case $n=3 m+2$ can be analyzed in a similar way.

The special case $n=4$ of this theorem is a counterpart to the theorem of Radon (1921) about points in the plane.

\section{Maximal Depth for $d \geq 3$}

We have proved that for arrangements $\mathcal{A}_{n}$ in $\mathbb{R}^{1}$ the maximal depth (2.1) is at least $\lceil n / 2\rceil$, whereas Theorem 1 showed that in $\mathbb{R}^{2}$ the lower bound is $\lceil n / 3\rceil$. Moreover, in $\mathbb{R}^{0}$ we trivially obtain $\lceil n / 1\rceil=n$. All these lower bounds are sharp. This would suggest the lower bound $\lceil n /(d+1)\rceil$ for any $d$.

More support for this possibility comes from the similar notion of location depth, where for any configuration of $n$ points in $\mathbb{R}^{d}$ the maximal depth is at least $\lceil n /(d+1)\rceil$. To our knowledge this result was first obtained in $\mathbb{R}^{2}$ by Neumann (1945), and in the case $d \geq 3$ by Rado (1946). The result was independently discovered by Birch (1959), and in statistics by Donoho and Gasko (1992). Each of these proofs makes use of Helly's theorem or the related results of Radon (1921) and Carathéodory (see, e.g., Danzer et al., 1963). In Edelsbrunner (1987, pages 63-66) a point with location depth at least $\lceil n /(d+1)\rceil$ is called a centerpoint, hence each configuration has a centerpoint.

We now conjecture that each arrangement contains a centerpoint as well. 
Conjecture 1. For any arrangement $\mathcal{A}_{n}$ in $\mathbb{R}^{d}$ with $d \geq 3$ it holds that

$$
\max _{\boldsymbol{\theta}} \operatorname{depth}\left(\boldsymbol{\theta}, \mathcal{A}_{n}\right) \geq\left\lceil\frac{n}{d+1}\right\rceil .
$$

Remark. There cannot be a better lower bound, since we can consider hyperplanes $h_{i}$ whose duals are $n$ different points on the moment curve $\left\{\left(t, t^{2}, \ldots, t^{d}\right) ; 0<t<\infty\right\}$.

Our attempts at generalizing the proof of Theorem 1 did not yield the desired result, nor did attempts to "dualize" the result for centerpoints of a configuration. Helly's theorem itself cannot be used for $\mathcal{A}_{n}$ since the relevant sets (determined by level surfaces of rotated versions of $\mathcal{A}_{n}$ ) are not convex. Also, the existing dual forms of Helly's theorem (see, e.g., Goodman and Pollack, 1982) yield hyperplanes instead of points.

We can also formulate a generalization of Theorem 3:

Conjecture 2. Any set $H_{n}$ of $n$ hyperplanes in $\mathbb{R}^{d}$ can be partitioned into $k=\lceil n /(d+$ 1) $\rceil$ subsets $I_{1}, \ldots, I_{k}$ such that $\bigcap_{j=1}^{k} C\left(I_{j}\right) \neq \varnothing$.

Here $C\left(I_{j}\right)$ stands for $\left\{\boldsymbol{\theta} \in \mathbb{R}^{d} ; \operatorname{depth}\left(\boldsymbol{\theta}, I_{j}\right) \geq 1\right\}$. Note that Conjecture 2 would imply Conjecture 1 , because a point $\boldsymbol{\theta}$ in $\bigcap_{j=1}^{k} C\left(\bar{I}_{j}\right)$ must satisfy

$$
\operatorname{depth}\left(\boldsymbol{\theta}, H_{n}\right)=\operatorname{depth}\left(\boldsymbol{\theta}, \bigcup_{j=1}^{k} I_{j}\right) \geq \sum_{j=1}^{k} \operatorname{depth}\left(\boldsymbol{\theta}, I_{j}\right) \geq k .
$$

In fact, Conjecture 2 has the same form as the theorem of Tverberg (1966) for a configuration of $n$ points in $\mathbb{R}^{d}$, if we read $C\left(I_{j}\right)$ as the convex hull instead of the contractible hull. Tverberg used this result to give an alternative proof of the Neumann-Rado theorem about the existence of a point with location depth $\geq k$.

Up to now we have considered arbitrary arrangements where it is possible that some hyperplanes are parallel or even coincide, or that all of them pass through the same point. For the next result we assume that $\mathcal{A}_{n}$ is in general position, meaning that any $d$ hyperplanes intersect in exactly one point in $\mathbb{R}^{d}$, and that any point in $\mathbb{R}^{d}$ lies on at most $d$ hyperplanes. (Hence no two hyperplanes can be parallel, and no $d+1$ hyperplanes can be concurrent.) Then we have the following upper bound, for all values of $d$.

Theorem 4. If the arrangement $\mathcal{A}_{n}$ in $\mathbb{R}^{d}$ is in general position, then

$$
\max _{\boldsymbol{\theta}} \operatorname{depth}\left(\boldsymbol{\theta}, \mathcal{A}_{n}\right) \leq\left\lfloor\frac{n+d}{2}\right\rfloor .
$$

Proof. Since $\mathcal{A}_{n}$ is in general position, the maximal depth (2.1) is reached in a point $\boldsymbol{\theta}$ that lies on exactly $d$ hyperplanes. (The case where $\mathcal{A}_{n}$ contains fewer than $d$ hyperplanes is trivial.) Note that $\operatorname{depth}\left(\boldsymbol{\theta}, \mathcal{A}_{n}\right)$ equals $(1.1)$, where the minimum ranges over all $\mathbf{u} \neq \mathbf{0}$ such that $\mathbf{u}$ (and hence also $-\mathbf{u}$ ) is not parallel to any hyperplane. Now consider the arrangement $\mathcal{A}_{n-d}$ obtained by removing the $d$ hyperplanes passing through $\boldsymbol{\theta}$. Then $n \operatorname{cross}\left(\boldsymbol{\theta}, \mathbf{u}, \mathcal{A}_{n-d}\right)+n \operatorname{cross}\left(\boldsymbol{\theta},-\mathbf{u}, \mathcal{A}_{n-d}\right)=n-d$ since each 
hyperplane in $\mathcal{A}_{n-d}$ is crossed by either $\operatorname{ray}_{\theta}(\mathbf{u})$ or $\operatorname{ray}_{\theta}(-\mathbf{u})$, but never both. Therefore $\min \left\{n \operatorname{cross}\left(\boldsymbol{\theta}, \mathbf{u}, \mathcal{A}_{n-d}\right), n \operatorname{cross}\left(\boldsymbol{\theta},-\mathbf{u}, \mathcal{A}_{n-d}\right)\right\} \leq\lfloor(n-d) / 2\rfloor$. Since this holds for all admissible $\mathbf{u}$ we find $\operatorname{depth}\left(\boldsymbol{\theta}, \mathcal{A}_{n-d}\right) \leq\lfloor(n-d) / 2\rfloor$ hence $\operatorname{depth}\left(\boldsymbol{\theta}, \mathcal{A}_{n}\right) \leq$ $\lfloor(n-d) / 2\rfloor+d=\lfloor(n+d) / 2\rfloor$.

\section{Computation}

Computing the depth of some $\boldsymbol{\theta} \in \mathbb{R}^{d}$ relative to an arrangement $\mathcal{A}_{n}$ is in general nontrivial. Rousseeuw and Hubert (1999) and Rousseeuw and Struyf (1998) constructed exact algorithms using $\mathrm{O}\left(n^{d-1} \log n\right)$ time. Since this takes too long for large $n$ and/or large $d$, the latter paper also proposes an approximate algorithm. We do not know whether a faster exact algorithm is possible.

For statistical applications we are mainly interested in finding the point(s) with maximal depth, i.e., the deepest point(s). As of yet, we only have a very naive algorithm for finding deepest points, which goes as follows. Any deepest point belongs to $d$ hyperplanes. We might thus compute all intersections of $d$ hyperplanes (in $\mathrm{O}\left(n^{d}\right)$ time) and determine the depth of each intersection point (in $\mathrm{O}\left(n^{d-1} \log n\right)$ time), yielding an overall computation time of $\mathrm{O}\left(n^{2 d-1} \log n\right)$. For $d=2$ we can lower the time complexity to $\mathrm{O}\left(n^{3}\right)$ (see Rousseeuw and Hubert, 1999) since the depth of a point can be computed in $\mathrm{O}(n)$ time after sorting the lines by decreasing slope. However, intuitively, we would expect that the deepest point in $d=2$ be computable in $\mathrm{O}\left(n^{2}\right)$ time or less.

The naive algorithms are too slow for routine use in statistical applications. By the duality (1.2), a deepest point in an arrangement corresponds to a regression fit yielding the most balanced summary of the data. For data analysis it is therefore of major importance to obtain algorithms that can deal with larger $n$ and $d$ in feasible time. We hope that this paper will inspire specialists of computational geometry to construct more efficient algorithms. We are optimistic since the computation of several other regression methods has been dramatically improved by techniques of computational geometry. For instance, the Theil (1950) line was originally computed by an $\mathrm{O}\left(n^{2}\right)$ algorithm but can now be computed in $\mathrm{O}(n \log n)$ time by algorithms of Cole et al. (1989), Dillencourt et al. (1992), and Matoušek (1991a). Randomized O $n \log n)$ algorithms for the repeated median slope estimator of Siegel (1982) were constructed by Matoušek et al. (1991). Finally, the least median of squares line (Rousseeuw, 1984) would naively take $\mathrm{O}\left(n^{3}\right)$ time to compute, but can now be found in quadratic time by the algorithm of Edelsbrunner and Souvaine (1990). Fast randomized algorithms for the least median of squares line were recently developed by Mount et al. (1997).

In the context of location depth in a configuration of points, several algorithms have been proposed for finding the deepest point (Matoušek, 1991b; Gil et al., 1992). Unfortunately, implementations of these algorithms are not yet available. Clarkson et al. (1996) gave a fast Monte Carlo algorithm for approximating center points of a configuration.

\section{Acknowledgments}

The authors are grateful to K. Clarkson, G. Koshevoy, and the reviewers for helpful comments which improved the presentation. 


\section{References}

Birch, B.J. (1959), On $3 N$ points in a plane, Proceedings of the Cambridge Philosophical Society, 55, $289-293$.

Clarkson, K.L., Eppstein, D., Miller, G.L., Sturtivant, C., and Teng, S.-H. (1996), Approximating center points with iterated Radon points, International Journal on Computational Geometry and Applications, 6, 357-377.

Cole, R., Salowe, J.S., Steiger, W.L., and Szemerédi, E. (1989), An optimal-time algorithm for slope selection, SIAM Journal on Computing, 18, 792-810.

Danzer, L., Grünbaum, B., and Klee, V. (1963), Helly's theorem and its relatives, Proceedings of the American Mathematical Society Symposium on Convexity, Seattle, pages 101-180.

Dillencourt, M.B., Mount, D.M., and Netanyahu, N.S. (1992), A randomized algorithm for slope selection, International Journal on Computational Geometry and Applications, 2, 1-27.

Donoho, D.L., and Gasko, M. (1992), Breakdown properties of location estimates based on halfspace depth and projected outlyingness, The Annals of Statistics, 20, 1803-1827.

Edelsbrunner, H. (1987), Algorithms in Combinatorial Geometry, Springer-Verlag, Berlin.

Edelsbrunner, H., and Souvaine, S. (1990), Computing least median of squares regression lines and guided topological sweep, Journal of the American Statistical Association, 85, 115-119.

Gil, J., Steiger, W., and Wigderson, A. (1992), Geometric medians, Discrete Mathematics, 108, 37-51.

Goodman, J.E., and Pollack, R. (1982), Helly-type theorems for pseudoline arrangements in $P^{2}$, Journal of Combinatorial Theory, Series A, 32, 1-19.

Lo, C.-Y., Matoušek, J., and Steiger, W. (1994), Algorithms for ham-sandwich cuts, Discrete and Computational Geometry, 11, 433-452.

Matoušek, J. (1991a), Randomized optimal algorithm for slope selection, Information Processing Letters, 39 , 183-187.

Matoušek, J. (1991b), Computing the center of planar point sets, in Discrete and Computational Geometry: Papers from the DIMACS Special Year, J.E. Goodman, R. Pollack, W. Steiger, eds., American Mathematical Society, Providence, RI.

Matoušek, J., Mount, D.M., and Netanyahu, N.S. (1998), Efficient randomized algorithms for the repeated median line estimator, Algorithmica, 20, 136-150.

Mount, D.M., Netanyahu, N.S., Romanik, K., Silverman, R., and Wu, A.Y. (1997), A practical approximation algorithm for the LMS line estimator, Proceedings of the 8th Annual ACM-SIAM Symposium on Discrete Algorithms, pages 473-482.

Neumann, B.H. (1945), On an invariant of plane regions and mass distributions, Journal of the London Mathematical Society, 20, 226-237.

Rado, R. (1946), A theorem on general measure, Journal of the London Mathematical Society, 21, 291-300.

Radon, J. (1921), Mengen konvexer Körper, die einen gemeinsamen Punkt enthalten, Mathematische Annalen, 83, 113-115.

Rousseeuw, P.J. (1984), Least median of squares regression, Journal of the American Statistical Association, 79, 871-880.

Rousseeuw, P.J., and Hubert, M. (1999), Regression depth, Journal of the American Statistical Association, 94, June issue.

Rousseeuw, P.J., and Ruts, I. (1996), AS 307: Bivariate location depth, Applied Statistics, 45, 516-526.

Rousseeuw, P.J., and Struyf, A. (1998), Computing location depth and regression depth in higher dimensions, Statistics and Computing, 8, 193-203.

Siegel, A.F. (1982), Robust regression using repeated medians, Biometrika, 69, 242-244.

Theil, H. (1950), A rank-invariant method of linear and polynomial regression analysis, Nederlandse Akademie Wetenschappen Series A, 53, 386-392, 521-525, 1397-1412.

Tukey, J.W. (1975), Mathematics and the picturing of data, Proceedings of the International Congress of Mathematicians, Vancouver, volume 2, pages 523-531.

Tverberg, H. (1966), A generalization of Radon's theorem, Journal of the London Mathematical Society, 41, $123-128$

Received December 1, 1997, and in revised form June 6, 1998. 